\title{
Design and Implementation of Modernised Dental Chair using Voice Recognition Control Circuit
}

\author{
R. Puviarasi, A. Greeshma
}

\begin{abstract}
Generally, in hospitals the dental chair can be operated forward/backward or upward/downward according to the treatment for the patients which is operated by human. Sometimes the chair will not function properly due to piston rust and over weighted patient and the dentist may have pain in the legs due to continuous operation of the chair. To overcome these issues, planning to design a voice recognition dental chair for the doctors in hospitals. This project describes the design of a smart, motorized, voice controlled dental chair. The voice command is given by the dentist/human, sensor recognizes the voice and sends the command to the Arduino. This voice command is converted to string and it is responsible for movement of chair. The intelligent dental chair is designed in such a way that it can be controlled easily by the doctor and has an advantage is the low cost design. This system was designed and developed to avoid wasting the energy and time of the doctor.
\end{abstract}

Keywords - Voice recognition, Arduino, Bluetooth module, ultra-sonic sensor, Pressure gauge, Pneumatic Cylinder, Solenoid Valve, Relay.

\section{INTRODUCTION:}

This model is conceived an idea to ease [1] the work of doctors who are using dental chair which consists of piston. Using voice initiated innovation for controlling the movement of the dental seat is to demonstrate that it can be a special idea that would stand separated from whatever is left of the normal undertakings. The utilization of this new innovation in conjunction with a mechanical framework keeping in mind the end goal to rearrange regular daily existence and it would start enthusiasm for a consistently developing present day society [2]. The problem is that sometimes the chair will not function properly due to piston rust and over weighted patient so the dentist may have pain in the legs due to continuous operation of the chair. So a voice controlled dental seat is produced which will work naturally on the charges from the client for development reason [3] and which is not available commercially [4]. The cost adequacy of the framework makes it reasonable for the specialists to utilize [5]. The system has a simple design [6]. The programmed discourse acknowledgment framework composed in this paper enables the dental seat to comprehend talked words inputted by a mike [7] and the dental seat moves as indicated by the given bearings [8]. The client can just control the dental seat by his voice utilizing particular summons to the forward, backward, upward and downward. The ultrasonic sensor is the device used to sense the obstacle back to the dental chair [9]. The

Revised Manuscript Received on July 18, 2019.

R. Puviarasi, ECE Department, Saveetha School of Engineering, Saveetha Institute of Medical and Technical Sciences, Chennai, India. Saveetha Institute of Medical and Technical Sciences, Chennai, India.
A. Greeshma, ECE Department, Saveetha School of Engineering,

module perceives the voice and sends control messages to the microcontroller. The proposed discourse acknowledgment based dental chair operation allows dentist to control the dental chair easily without the need to use hands [10]. The movement of the dental chair is controlled by the motors and motor drivers connected to the chair [11] and drive framework which comprises of microcontroller and motor driving [12]. On getting the Signal the microcontroller coordinates the motors through the control circuit. In this, two DC brushless motors are utilized for controlling the chair independently. The code is written in Arduino such that the speed of the motors is controlled by using output pins of Arduino [13]. A speech reorganization system is easy to use programmable speech reorganization circuit that is the system to be prepared the words the client needs the circuit to perceive. A speed controller works by changing the normal voltage send to the motor [14]. Specifically, this is valuable for the dental specialists where they can move their dental seat in their own ways, with no outsider's assistance or support [15]. Speech technology is used to reduce physical effort of the dentist a voice module is utilized to perform voice action. [16]. This paper develops a voice controlled dental chair which uses the voice command as the interface. The system has been planned and executed in a financially savvy way so that if our project is popularized the destitute clients in creating nations will profit by it. We trust our venture will be relevant for some valuable operation and give some progression in innovation, and most vital this may give some assistance to the dentists. The use of the microcontroller makes the system to work independent, without the need of utilizing any PCs and furthermore empowers a minimal effort, little size, and low vitality utilization plan. This paper is based on Voicecontrolled dental chair configuration in light of versatile stages, by methods for Bluetooth innovation, outline and usage of remote control arrangements.

\section{MATERIALS}

Arduino: Arduino is an open source platform for making electronic projects. It is an instrument for making devices that can sense and control more of present world. It is used mostly in present day developing techniques. It is a microcontroller development board for writing software for the hardware circuit. The programming language used for Arduino is c programming language. The Arduino is the

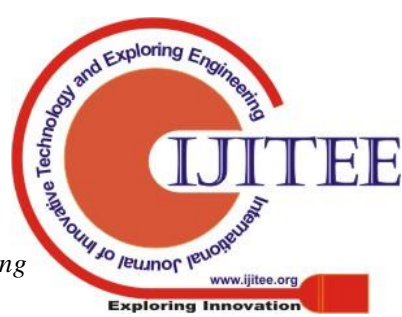




\section{DESIGN AND IMPLEMENTATION OF MODERNISED DENTAL CHAIR USING VOICE RECOGNITION CONTROL CIRCUIT}

core part of the voice controlled dental chair. It acts as an interface between the voice recognition unit and the motor driver circuit

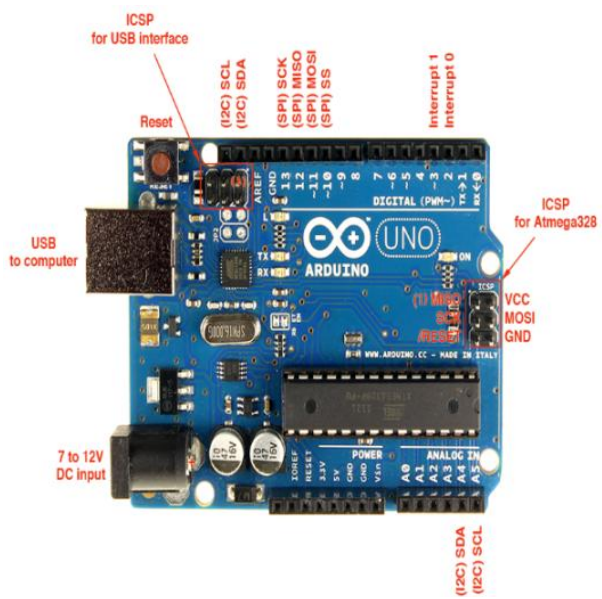

Fig 1: Arduino board

Voice Recognition Module: Voice recognition is that the method of changing the speech signal to a sequence of word by suggests that of associate algorithmic program enforced as a program that is additionally referred to as Automatic speech recognition (ASR). Essentially speaker freelance systems square measure most generally used as a result of voice coaching isn't used there. Speech recognition is classed as connected word recognition and isolated word recognition. Therefore, the speech recognition is nothing however the pattern matching supported training and recognition [2].

Motors: This circuit is in charge of the DC motor turn. The circuit comprises of four transfers to move required way and furthermore to stop development. [17]

Relay: A relay is an electro-mechanical switch capable of being remotely actuated or controlled. These are used when several circuits may be controlled by one signal or where it is necessary to control a circuit by a separate low-power signal.

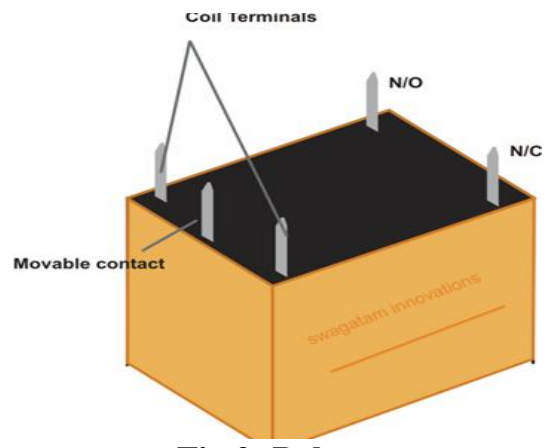

Fig 2: Relay

Pneumatic Cylinder: These are also known as aircylinders which use the power of compressed gas to produce a force to other components in a linear motion.

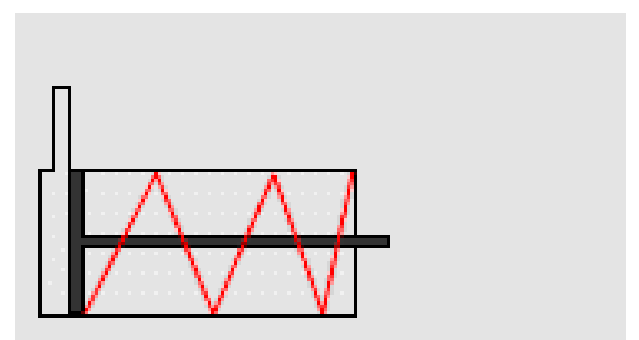

Fig 3: Pneumatic cylinder

\section{METHODOLOGY}

This paper deals with the voice controlling mechanism of dental chair. It consists of two parts they are hardware and software. The hardware architecture consists of an embedded system which consists of Arduino Uno board, voice recognition module, a Bluetooth Module, Driven motor. It works on the commands given by the dentist. The voice modules are converted into machine understandable codes by attaching an Arduino. Arduino acts as an intermediate between the software and the hardware modules. Pneumatic cylinders are responsible for the movement of the chair. The chair moves up and down as the pressure is accumulated on the chair by the release of the gas. A pressure gauge is attached to the pneumatic cylinder and a solenoid valve is attached to it. All these work in a synchronous manner. If they don't work in synchronous manner the movement of chair is not in a linear manner. Pneumatic cylinders extend and retract a piston rod to provide a push or pull force to drive the external load along a linear manner. The desired command given by the user is sent through microphone. The given input is sent to a voice recognition module. The voice command is changed over to a variety of string is passed to Arduino board associated to it. An Arduino is set up to the chair. Arduino converts these instructions into certain commands that can be recognized by the motors. This controls the movement and direction of wheel chair through motor. Arduino decides the movement of the motors. Primarily, Voice module is trained with some commands. The user commands are compared with the trained commands. The Arduino matches the commands and gives the information to the motors. According to the decisions made by the Arduino, the motors works. These commands are given to the dental chair using electrical signs which are utilized to drive the motors.

\section{BLOCK DIAGRAM}

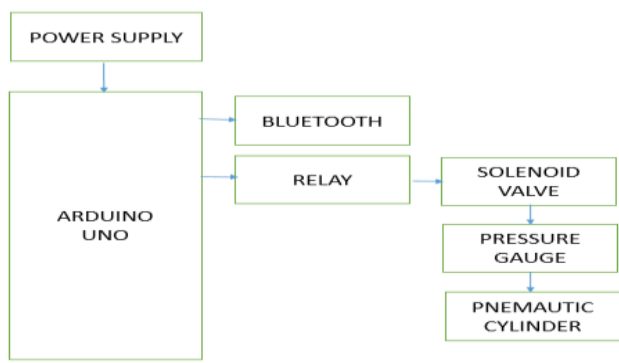

Fig 4: Block diagram for dental chair circuit 


\section{WORKING PRINCIPLE}

In this project, it is implemented to Arduino controller which is mainly used for dental chair in dental hospitals. It is controlled by patient chair using Pneumatic cylinder and Bluetooth is used to transmit the data. When I say command to the Bluetooth that time the controller receive the data and the relay will automatically on the solenoid valve and it release the pressure. So the chair will change automatically with given respective command.

The different directions of motions possible are: up, down, front, back and stop. In achieving the task, the controller is loaded with program using the Arduino programming language and Arduino development environment. In this project we are designing for only two different directions of motions: upward and downward.

\section{RESULT}

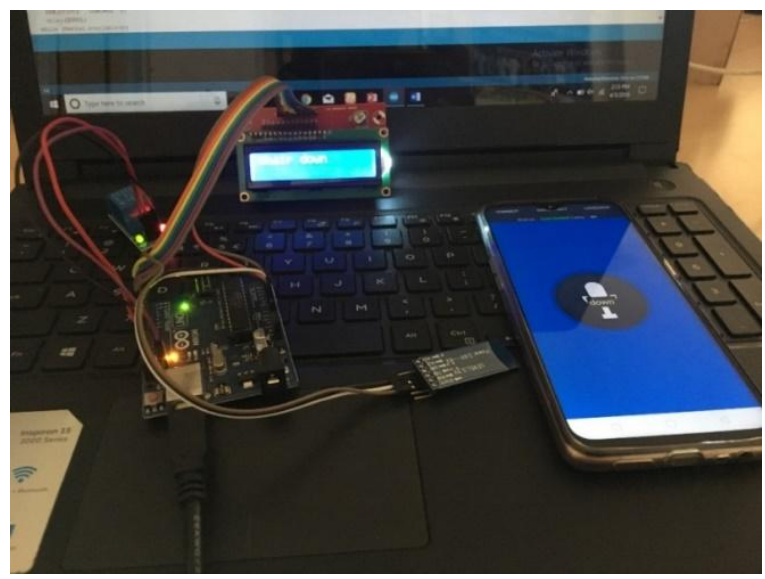

Fig-5: Output

In the above given figure-5 it shows the result, when the user says command DOWN the circuit responds to it and the chair moves DOWNWARD and that can be seen in the LCD display. Similarly, for the UP command also it works the way.

\section{CONCLUSION}

For the most part in hospitals the dental seat can be worked forward, reverse or upward as indicated by the treatment for the patients which is worked by human. Once in a while the seat will not work legitimately because of cylinder rust and over weighted patient and the dental specialist may have torment in the legs because of consistent operation of the seat. To beat these issues, it is intending to outline a voice acknowledgment dental seat for the hospitals. This task portrays the plan of a savvy, mechanized, voice controlled dental seat. This project has more advantages compare to conventional methods. It includes, it is low cost, reduces the effort of the dentist, user friendly, easy to design.

\section{REFERENCES:}

1. Smart Electronic Wheelchair Using Arduino and Bluetooth Module. Deepak Kumar Lodhi, PrakshiVats, Addala Varun, Prashant Solanki, Ritakshi Gupta, Manoj Kumar Pandey, Rajat Butola, IJCSMC, Vol. 5, Issue. 5, May 2016.
2. Design of Voice Controlled Smart Wheelchair. Ali A. Abed (MIEEE), International Journal of Computer Applications, December2015.

3. Voice Controlled Wheelchair using Arduino. Apsana.S, Renjitha G Nair, IARJSET, August 2016.

4. Voice Controlled WheelChair Using Arduino. Kharka Bahadur Rai, Jeetendra Thakur, Nirmal Rai, IJSTM, June 2015.

5. Voice Controlled Wheelchair Using AVR, Prof. D.S. Nikam, Joshi Gauri, Shinde Mohini, Tajanpure Mohini, Wani Monika, IJMTER, 2014.

6. Voice based Wheelchair for Physically Challenged. Ravi Teja Ch. V, P. Shekar, S. Hari Prasad Reddy, S. Roja, Y. Bhargavi, IJNIET, March 2015.

7. Designing and Modeling of Voice Controlled Wheel Chair Incorporated with Home Automation. Anoop.K. J, Inbaezhilan, Satish raj, Ramaseenivasan, CholaPandian, IJAREEIE, April 2014.

8. Voice Operated Wheelchair for physically challenged People., Smita U. Upase, A. K. Joshi, IJASET, August 2016.

9. Voice controlled Autonomous Wheelchair. Krishna Pal Tiwari, Mr Kranti Kumar Dewangan, IJSR, April 2015.

10. Design and Development of Smart Wheelchair using Voice Recognition and Head Gesture Control System. Srishti1, Prateeksha Jain, Shalu, Swati Singh, IJAREEIE, May 2015.

11. Voice controlled intelligent wheel chair. Takeshi saitoh, conference paper, October 2014.

12. Wheel chair using Voice Recognition Systems for Paraplegics. Angel Mercy.T, Jeshua Linu. J, IJARET, April June 2017.

13. Low cost Self-Assistive Voice controlled Technology for Disabled People. R. Puviarasi, Mritha Ramalingam, Elanchezhian Chinnavan, IJMER, Jul.-Aug. 2013.

14. Touchpad and Voice Command Based Wheelchair 1Dipanjali P. Panchal, 2Priya B. Parmar, 3Nidhi S. Ganasva, 2017 IJEDR.

15. A Voice Controlled Wheel Chair Prototype for a Medically Challenged. Rahul Agarwal1, Akram Siddiqui2, Kuvendra Singh3, Arjun Solanki4, Lavit Gautam5, IJETT April 2016.

16. Voice Operated Wheel Chair. Jayesh K. Kokate1, A. M. Agarkar2, IJRET Feb 2014. 